\title{
ESTUDO SOBRE OS BENEFÍCIOS DO BIM NA INTEROPERABILIDADE DE PROJETOS
}

\section{ARTIGO ORIGINAL}

BARROS, Fernando da Costa ${ }^{1}$

MELO, Humberto Coelho de ${ }^{2}$

BARROS, Fernando da Costa. MELO, Humberto Coelho de. Estudo sobre os benefícios do BIM na interoperabilidade de projetos. Revista Científica Multidisciplinar Núcleo do Conhecimento. Ano 05, Ed. 01, Vol. 08, pp. 74-91. Janeiro de 2020. ISSN: 2448-0959, Link de acesso: https://www.nucleodoconhecimento.com.br/engenhariacivil/interoperabilidade-de-projetos

\section{RESUMO}

Como forma de otimizar os processos nos setores da Arquitetura, Engenharia e Construção ( $A E C$ ), o desenvolvimento de projetos se tornou fragmentado para a diminuição de custos e tempo de trabalho. Entretanto, com a inconsistência de projetos decorrentes da incompatibilidade entre softwares, inúmeras atividades foram adaptadas, gerando retrabalhos que impactam diretamente no cronograma de projetos. Com a utilização do conceito BIM (Building Information Modeling), o sistema construtivo se tornou uma eficiente alternativa no processo de interoperabilidade projetos, possibilitando a exequibilidade construtiva na coordenação de sistemas. A metodologia proposta visa contextualizar os conceitos BIM, através referências bibliografias, associando aos benefícios do conceito aplicado: na gestão de projetos,

${ }^{1}$ Bacharel em Administração pela PUC-MG e Graduando em Engenharia Civil pelo IFMG.

2 Mestre em Construção Civil pela UFMG, Especialista em Gestão de Projetos pela Fundação Dom Cabral e em Licenciamento Ambiental pela PUC-MG.

Disponível em: https://www.nucleodoconhecimento.com.br/engenharia-civil/interoperabilidade-deprojetos 
no processo de modelagem paramétrica e na interoperabilidade de projetos do setor público e privado, tendo como fundamentos analisar o cenário atual BIM no Brasil, sob a ótica da regulamentação e difusão da ferramenta. Este estudo se justifica em função de mudanças de tecnologias ocorridas a favor da melhoria do gerenciamento de informações, de investimentos, e de transparência no setor. Contudo, foi possível verificar os benefícios da utilização do conceito BIM durante o estudo na otimização de projetos; entretanto, no setor privado verificou-se que o método pouco difundido, mediante ao alto custo assim como a necessidade mão de obra qualificada; no setor público ainda necessita-se de informações acerca de modelos implementados nos órgãos governamentais que sejam de acesso público.

Palavras-chave: Interoperabilidade, projetos, BIM.

\section{INTRODUÇÃO}

Com a modernização do mercado econômico, muitos setores foram impactados com o desenvolvimento de novas tecnologias como forma de diferencial competitivo, a fim de aprimorar e desenvolver atividades com maior qualidade ao menor custo. Entre os setores econômicos, os setores da Arquitetura, Engenharia e Construção (AEC) sofreram um grande impacto com a modernização, beneficiando-se de técnicas construtivas, como meio de otimização de projetos e de recursos.

Para que a tecnologia seja benéfica para estes setores, torna-se necessário que os projetos sejam planejados e executados de forma coordenada e efetiva. Entretanto, para Thomaz (2001), a construção brasileira envolve inúmeros problemas decorrentes de falhas na execução de projetos, assim como na falta de harmonização entre os mesmos.

Como forma de propor novas soluções para o planejamento e execução de projetos na AEC, o BIM (Building Information Modeling) surgiu como uma forma de integralizar as informações, sendo instituído por múltiplos profissionais, que estejam envolvidos em um único projeto, afim de propor resultado mediante a sincronização de informações de forma eficiente, favorecendo a melhoria na comunicação entre a

Disponível em: https://www.nucleodoconhecimento.com.br/engenharia-civil/interoperabilidade-deprojetos 
cadeia produtiva, desde projetistas até o cliente. Eastman et al. (2013), relatam que BIM visa propor soluções na elaboração de projetos, otimizando os processos do setor, aumentando a qualidade e o desempenho da edificação, permitindo uma representação gráfica dos sistemas, desde a fase da concepção, da construção até o processo de desmontagem ao fim do ciclo de vida do empreendimento.

Com a utilização do BIM, o procedimento de compatibilização de projetos se tornou eficiente, mediante compartilhamento de informações entre diferentes plataformas por meio do formato IFC (Industry Foundation Classes) sem que haja perda de dados mediante fragmentação, possibilitando o reagrupamento de informações das diferentes áreas de múltiplos agentes. Eastman et al. (2013) afirmam que a plataforma IFC foi desenvolvida por meio de um conjunto de dados gerados de forma consistente para representação dos componentes de um edifício, de modo consistente, com o objetivo de permitir o compartilhamento de informações entre diferentes softwares empregados na AEC.

Através do planejamento de projetos, vinculados à tecnologia proposta pelo BIM, muitos setores serão beneficiados, incluído o setor público, reaproveitando de modo eficiente os recursos e prestação de contas de forma transparente, evitando superfaturamento de projetos e uso inadequado de recursos materiais e financeiros, evitando falhas construtivas,de modo que seja possível prever a implantação de sistemas instalados de forma não adequada ou mal dimensionados, além de outros prejuízos como compatibilização de informações vindas de outros setores. Entretanto, para que haja a expansão do BIM no Brasil se torna primordial a implantação de diretrizes e de padrões que visem regulamentar a adoção do setor privado e público.

A escolha do tema em questão, assim como na formulação para o desenvolvimento do estudo, se deve ao fato da necessidade de analisar o avanço dos sistemas construtivos da construção civil, juntamente com os empreendimentos do setor público sobre os impactos da adesão da modelagem da construção, para melhor conhecimento sobre o tema, por meio do embasamento teórico. 
Neste contexto, a importância deste estudo consiste em uma análise bibliográfica sobre os fundamentos, aplicações e conceitos do BIM no setor da AEC, destacando os benefícios para gestão do planejamento de projetos, a interoperabilidade entre projetos, demonstrando a situação do BIM no Brasil na adoção em projetos do setor privado e na Administração Pública.

\section{REVISÃO BIBLIOGRÁFICA}

O método científico ou "conhecimento científico não é apenas um produto resulte no processo de uma investigação, mas o desejo de permitir explicações sistemáticas que possam ser formuladas e questionadas mediante a apresentação de evidências empíricas e para discussão" (KÖCHE, 2015, p.29).

Este estudo consiste na análise sobre as produções bibliográfica que parte na compreensão da relevância do BIM para o setor, assim como a necessidade da migração de projetos para outras plataformas de projeção de modo a adequar-se as mudanças do cenário no construção civil brasileiro.

\subsection{PROJETO}

O projeto pode ser definido como "esforço temporário realizado como a finalidade de obtenção de um produto ou serviço, sendo que a natureza dos projetos pode indicar que eles têm um início e término definidos ou mediante a necessidade do cliente" (PMBOK, 2013, p.3).

Melhado apud Thomaz (2001) afirma que o trabalho de coordenação de projetos é considerado uma tarefa árdua e complexa, em que a eficiência dependerá, exclusivamente, da qualidade dos materiais e métodos utilizados na elaboração. Além disso, o projeto pode ser caracterizado, ainda, como um processo desenvolvido para um ramo específico da AEC.

A importância de uma gestão eficiente na elaboração de projetos pode ser justificado mediante a imagem 1, que descreve sobre a curva de projetos, sendo que o

Disponível em: https://www.nucleodoconhecimento.com.br/engenharia-civil/interoperabilidade-deprojetos 
planejamento elaborado de forma adequada se reverte, posteriormente, em benefícios, como: diminuição do tempo e do custo do empreendimento.

Figura 1 - Impactos do projeto no custo do empreendimento.

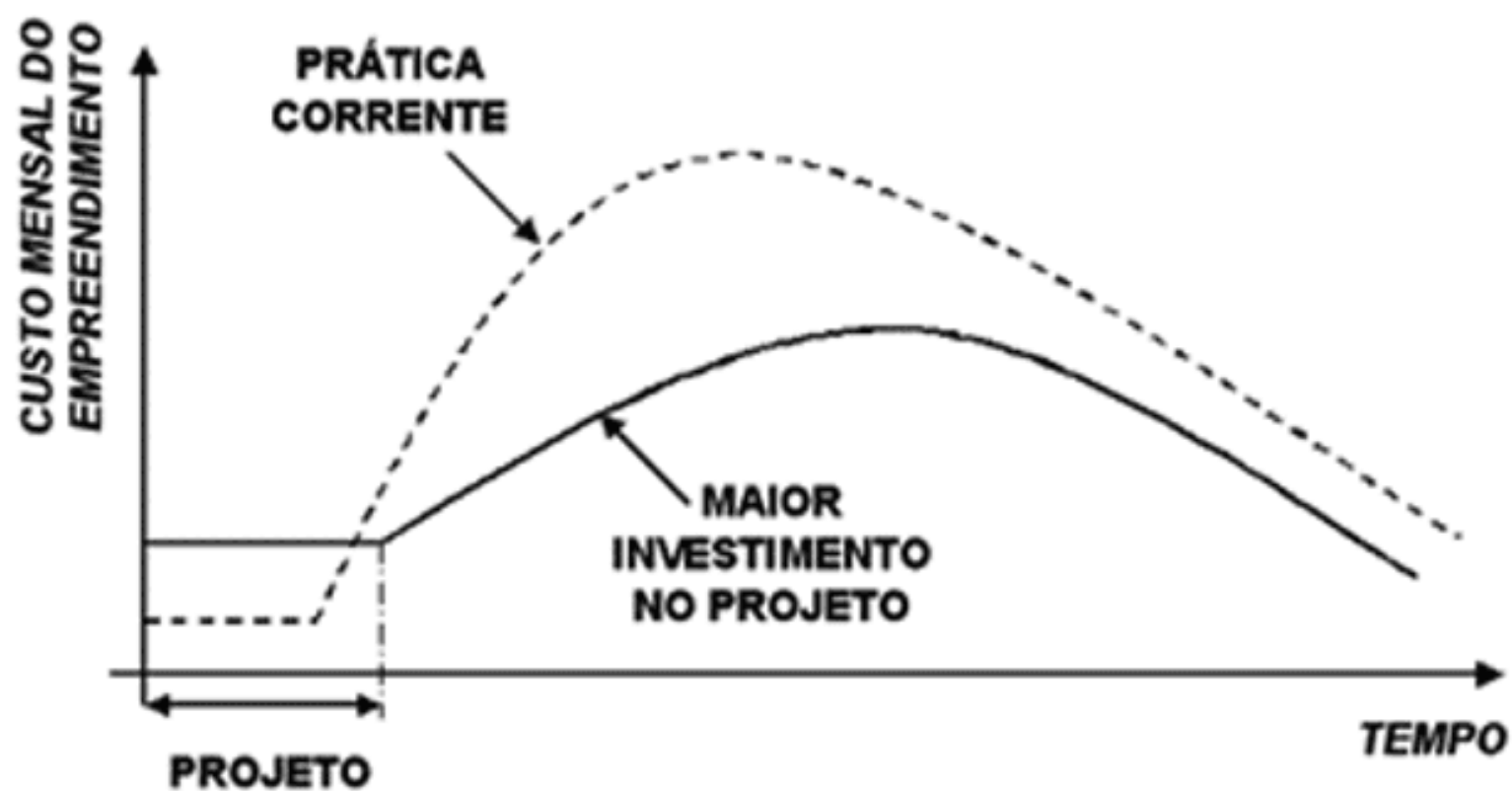

Fonte: Sul21, 2018.

Torna-se fundamental gerir adequadamente o desenvolvimento de projetos para os setores AEC, sendo importante avaliar que o ciclo de vida dos projetos, através dos sistemas construtivos empregados permitindo analisar e validar critérios definidos na fase de concepção, juntamente com os impactos econômicos, ambiental e social dos empreendimentos.

Thomaz (2001) destaca ainda que as principais dificuldades de coordenação de projetos consiste em:

- No início do projeto, em função da característica do empreendimento, a empresa construtora pode ou não estar contratada, ou seja, fica difícil agregar contribuições de engenheiros da produção; 
- A contratação dos projetistas de estrutura, fundações e sistemas prediais ocorre, normalmente, somente na fase de projeto préexecutivo, também fica difícil a contribuição destes profissionais na fase de concepção da obra;

- Falta de participação ativa da construtora ou de profissionais com visão da produção nas etapas iniciais de desenvolvimento de projetos;

- Ocorrência de modificações nos projetos acarretadas por exigências das municipalidades ou concessionárias de serviços públicos, ou mesmo por mudança de ideia do proprietário da obra (THOMAZ, 2001, p. 275).

O guia PMBOK (2013) ressalta que os projetos dentro de um programa são relacionados entre si, com o objetivo de alcançarem resultado em comum, além da capacidade de relacionar projetos entre clientes, gestores e fornecedores.

Com a utilização do BIM, o desenvolvimento de gerenciamento de canteiro de obras se torna mais eficiente, por meio da realocação e armazenamento de matéria-prima e equipamentos, permitindo o fácil acesso aos dados do projeto entre colaboradores, fornecedores e projetistas, de modo que por meio de um projeto estruturado, a construção se torna ágil, mediante processo de linha de montagem em que cada parte da construção é executada.

\subsection{BIM- BUILDING INFORMATION MODELING}

O conceito e a nomenclatura do BIM envolve a diversidade de conhecimentos de profissionais da AEC. Para Eastman et al. (2013), o conceito do BIM passou a ser difundindo no Brasil nas últimas décadas.

Os conceitos, as abordagens e metodologias que conhecemos hoje se identificam com o BIM têm cerca de trinta anos, e a terminologia do Building Information Model está em circulação há pelo menos quinze anos (EASTMAN et al., 2013, p.01).

Disponível em: https://www.nucleodoconhecimento.com.br/engenharia-civil/interoperabilidade-deprojetos 
Criado nos anos 1970, o BIM ganhou destaque nos anos seguintes como forma de propor novas soluções para o desenvolvimento de projetos, permitindo o suporte de múltiplas vistas de diferentes dados de modo contínuo em um único modelo de desenho, incluindo o 2D e 3D (EASTMAN et al, 2013). No Brasil, a modelagem da construção despontou por volta do ano de 2004, tendo o próprio termo BIM (Building Information Modelin) (SANTOS, 2017).

Segundo a empresa de Software AUTODESK (2019), a tecnologia BIM consiste em um processo de geração de informações de forma inteligente, através da modelagem tridimensional, capaz de que oferecer dados e conhecimentos aos profissionais AEC para o processo de planejamento, execução e gerenciamento de empreendimentos de forma mais eficiente.

Para Oliveira (2016), o uso da modelagem de informações permite agregar informações, que vão além do uso de representações em 3D, assim como na consolidação de informações por meio de um banco de dados, cronograma do projeto, representação gráfica, controle do tempo de mão de obra e quantificação, sejam geradas de forma automática como forma de minimizar falhas, testando alternativas, ensaios no que corresponde ao comportamento gerado por meio do modelo.

Karstila apud Thomas pondera que o desenvolvimento de sistemas inteligentes no campo da construção pode resolver diversos problemas como:

- atendimento às prescrições da normalização técnica, códigos de obras, leis e regulamentos;

- produção de anteprojetos e desenhos preliminares;

- seleção de soluções técnicas;

- diagnósticos de falhas nos projetos. (KARSTILA apud THOMAS, 2001, p.279).

Com a evolução das ferramentas computacionais, o BIM surgiu como uma forma de integração de informações de diversas disciplinas, sendo capaz de envolver múltiplos profissionais em uma mesma etapa do projeto, com o objetivo de proporcionar

Disponível em: https://www.nucleodoconhecimento.com.br/engenharia-civil/interoperabilidade-deprojetos 
resultados eficientes no decorrer da sincronização de informações. Segundo Moreira (2015), há um interesse crescente na indústria da construção civil no uso do BIM, em função das facilidades da gestão da informação, que permite a incorporação de dados consistentes desde a concepção da construção até as fases de operação e de manutenção.

Desse modo, "a tecnologia da modelagem da informação da construção proporciona a facilidade no desenvolvimento de trabalhos simultâneos de múltiplos projetos, permitindo o gerenciamento em todas as etapas do projeto como forma de evitar erros ou omissões" (EASTMAN et al., 2013, p.17).

Entre as vantagens do BIM, a maneira de previsibilidade de resultados, antes da estruturação de projetos na fase de desenvolvimento, proporciona informações vertentes na coordenação de trabalho, facilitando a integração entre os sistemas, proporcionando uma análise do ciclo de vida do projeto.

Para Andrade; Ruschel (2009, p.78): "o uso do BIM na atividade de projeto arquitetônico, facilita no processo de geração de quantitativos (aproximadamente 23\% dos arquitetos geram quantitativos a partir dos modelos BIM), seguido pela checagem de conflitos (cerca de $21 \%$ )".

Coma a adoção dos conceitos BIM nas organizações, projetistas podem investir mais tempo em planejamento e pesquisas da obra, como forma de evitar desperdícios e retrabalhos na execução. "Sendo que ao longo do ciclo de vida do projeto, a quantidade de dados e informações coletadas podem ser analisadas e distribuídas como relatórios para vários membros da equipe do projeto e outras partes interessadas" (PMBOK, 2013, p.58).

Mediante as descrições apresentadas é possível elencar que o BIM proporciona a modelagem paramétrica, permitindo o levantamento de matéria-prima, a interoperabilidade entre projetos, assim como na geração de simulações. 


\subsection{INTEROPERABILIDADE}

A interoperabilidade pode ser descrita como um processo que envolve muitas fases e com diferentes participantes, que necessitam de troca de informações ao longo de todo ciclo de vida do projeto. Porém, dificuldades na troca de informação em função de baixa interoperabilidade aparecem como fatores limitantes do uso do BIM no processo de projeto (ANDRADE; ROSCHEL, 2009).

Para a sobreposição de projetos é necessário conhecer as ferramentas que serão utilizadas, além das técnicas que irão auxiliar na coordenação entre as disciplinas de projetos, resultando na compatibilidade entre os projetos.

A interoperabilidade retrata, de modo geral, a necessidade de transmissão de dados entre diferentes ferramentas, de modo que seja possível a contribuição de diferentes especialistas e aplicações ao trabalho de equipe (EASTMAN et al., 2013).

Ainda, em Andrade; Roschel (2009, p.78), para que haja "uma boa interoperabilidade é de fundamental importância da implementação de um padrão de protocolo internacional de trocas de dados nos aplicativos e nos processos do projeto".

Um dos fundamentos do emprego BIM em projetos da AEC consiste na utilização ideal de modelo de projeto único para uma maior segurança e continuidade das informações, evitando redundância no processo. Todavia, houve situações que, ao exportar o modelo em IFC, todos os elementos eram armazenados com segurança e qualidade. $\mathrm{O}$ arquivo IFC permite que múltiplos projetistas se utilizem de diferentes plataformas de projetos sem que isso impeça o desenvolvimento do trabalho, seja em conjunto ou integrado. Andrade; Roschel (2009, p.78) destacam que o "principal protocolo usado hoje é o IFC, que é um modelo de dados do edifício baseado em objetos".

Para Andrade; Roschel (2009, p.80): " a passagem de dados entre aplicativos são utilizados arquivos baseados em diferentes formatos de trocas. Alguns destes 
aplicativos apresentam maior capacidade de interoperabilidade, outros se limitam às trocas internas".

\subsection{IFC- INDUSTRY FOUNDATION CLASS}

A interoperabilidade representa a necessidade de compartilhamento de dados e aplicações para múltiplos especialistas para a contribuição do trabalho. De modo geral, a interoperabilidade é baseada no intercâmbio entre formatos de arquivos, que intercambiam por meio da geometria (EASTMAN et al., 2013).

Um aspecto fundamental da interoperabilidade pode ser apresentado através da ferramenta IFC, que consiste em um modelo de ferramenta com código aberto, sendo possível trabalhar com qualquer aplicativo, tornando seu processo abstrato. Entidades de diferentes aplicativos podem ser combinadas e relacionadas de maneira única, de acordo com definições particulares (ANDRADE; ROSCHEL,2009).

Com forma de proporcionar a interoperabilidade de projetos desenvolvidos em diversas plataformas que adotam o conceito BIM, a organização sem fins lucrativos Building Smart desenvolveu um padrão a extensão de arquivos denominada Industry Foundation Class (IFC). O objetivo deste formato é permitir o compartilhamento de dados, entre todos os participantes, independentemente do software que eles utilizem (BUILDINGSMART, 2018). 
Figura 2 - Interoperabilidade entre projetos.

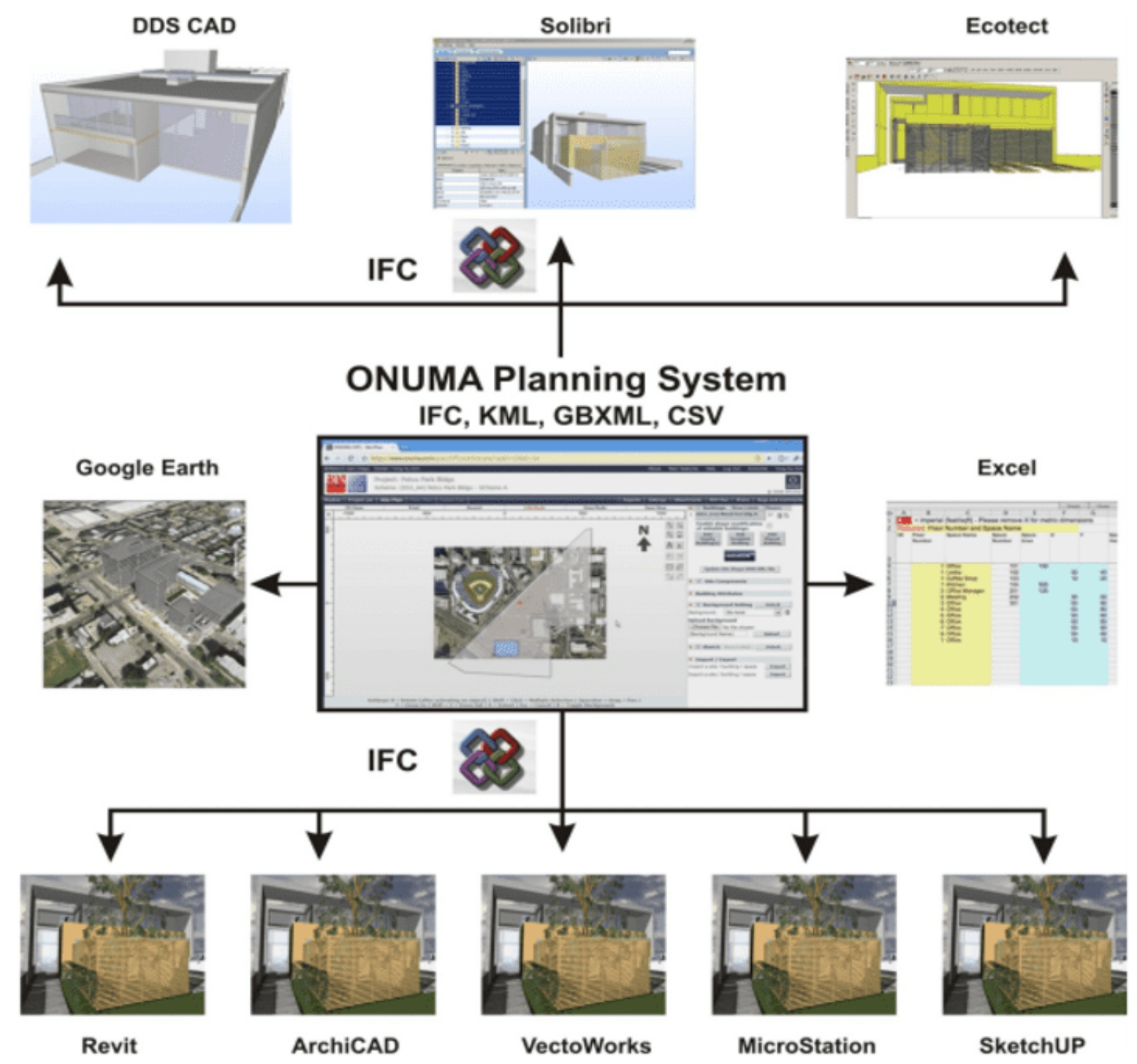

Fonte: ONUMA PLANNING SISTEM (2008) apud Andrade; Roschel (2009).

O IFC foi criado para representar dados consistentes da construção e realizar a troca entre os aplicativos utilizados nos projetos. Essa linguagem é focada em fornecer definições gerais dos modelos, tratamento das informações geradas e o ciclo de vida do empreendimento (EASTMAN et al., 2013).

O IFC pode ser definido como:

Disponível em: https://www.nucleodoconhecimento.com.br/engenharia-civil/interoperabilidade-deprojetos 
Termo usado para designar um esquema básico e um conteúdo de dados, composto de acordo com um padrão internacional, aberto e acessível ao público, para a estruturação e a troca de informações entre aplicativos computacionais voltados para a indústria da AEC (HAAGENRUD et al., 2007, apud ANDRADE; ROSCHEL,2009, p.83).

As propriedades que são usadas para a utilização dos padrões IFC se desenvolvem através de materiais, de propriedades contextuais, como: ventos, dados geológicos ou de clima, entre outros, destacando as relações existentes entre objetos e propriedades (Eastman et al., 2013).

Atualmente, são encontrados no mercado inúmeros softwares que integram ao conceito BIM, entre esses: Revit, ArchiCad, Bentley Architecture, VectorWorks, sendo que todos permitem a exportação com um formato de arquivos de dados de arquitetura aberta, uma linguagem comum para que haja troca entre os diversos modelos, conhecida como IFC (MUNDO...,2018).

Para Carvalho (2011), com a implantação do uso da modelagem BIM torna-se necessário a definição de um modelo de estrutura de dados urbanísticos vinculados ao modelo da edificação em projeto para possibilitar a conversão e uso de modelos BIM (com padronização IFC) e a garantia da segurança da informação.

\subsection{BIM EM OBRAS PÚBLICAS}

A indústria da construção civil nas brasileiras está entre as maiores do mundo, tornando-se responsável por $2 \%$ da indústria global, impactando na economia e no desenvolvimento do país. Com a adoção dos conceitos e ferramentas BIM se percebe, de imediato, resultados positivos no setor (Kassem e Amorim, 2015).

Com o crescimento do setor da $A E C$, surge a necessidade de uma gestão eficiente em projetos voltados para as obras públicas, para que seja possível elevar os setores em um novo patamar de desenvolvimento do país. Com a ausência do controle e fiscalização de projetos em obras públicas no Brasil ocasiona uma conjectura sobre o

Disponível em: https://www.nucleodoconhecimento.com.br/engenharia-civil/interoperabilidade-deprojetos 
uso dos recursos públicos, que contamina toda a sociedade, ocasionando uma econômica e social nas camadas populares brasileiras (PORTAL...,2018).

Santos (2017) apresenta o contexto geral do panorama brasileiro:

Ainda assim, o conceito de BIM como processo ainda não foi completamente assimilado pelos empresários do setor da construção. Com frequência ainda alta, depara-se com casos falhos de implantação de BIM. O prognóstico de fracasso, na maioria destes casos, é evidente para os iniciados, mas certamente não era para aqueles que investiram tempo e recursos significativos na expectativa de obter o salto qualitativo em seus resultados prometido pelo BIM. (SANTOS,2017, p.64).

Kassem e Amorim (2015) destacam que a implantação do BIM no setor público ocorre de maneira limitada se comparado com outras áreas acadêmicas, sendo que há uma das exceções, o Exército Brasileiro, por ser o pioneiro na implantação bem-sucedida do BIM para o planejamento e controle de suas operações. Em um contexto de Brasil, em função da dimensão territorial do país, as políticas de implementação e de desenvolvimento do BIM devem contar com o apoio da indústria.

Porwal e Hewage (2013) apud Barreto et al. (2016) destacam que:

...para uma melhor difusão do conceito no Brasil, seria muito importante o incentivo do setor público para a sua difusão. Porém apenas algumas iniciativas nacionais isoladas têm sido tomadas por alguns órgãos como a Petrobrás, o Departamento Nacional de Infraestrutura de Transportes (DNIT), o Exército Brasileiro, o Governo de Santa Catarina, o Instituto Nacional de Propriedade Industrial (INPI), para a implantação do BIM no mercado brasileiro. (BARRETO, 2016, p.02).

Kassem e Amorim (2015) apresentam que Santa Catarina foi um dos primeiros Estados a proporcionar edital para contratação de projetos BIM como forma de contratação, sendo que a partir de 2015 todos os projetos devem ser apresentados 
através desta forma e ainda devam ser regulamentados. A iniciativa consistia em desburocratização e o incentivo a automatização dos requisitos técnicos no processo de licitação por técnica e preço.

Entre os problemas decorrentes da expansão do BIM, no país, pode ser exposta a ausência de leis e decretos relativos ao uso do BIM,sendo queno país se encontra, atualmente, duas normativas importantes para a difusão e regularização do conceito nos setores AEC, entre essas, a ABNT NBR 15965 e o Decreto 9.377.

\subsection{NORMALIZAÇÃO DO BIM NO BRASIL}

A partir do ano de 2010, a Associação Brasileira de Normas Técnicas (ABNT) designou comissão para a normalização do BIM, classificando e identificando objetos das bibliotecas, sendo criadas desde então as seguintes normas:

ABNT NBR 15965-1:2011 Sistema de classificação da construção. Parte 1: Terminologia e estrutura.

ABNT NBR 15965-2:2012 Sistema de classificação da construção. Parte 2: Características dos objetos da construção.

ABNT NBR 15965-3:2014 Sistema de classificação da construção. Parte 3: Processos da construção.

ABNT NBR 15965-7:2012 Sistema de classificação da construção. Parte 7: Informação da construção. (ASSOCIAÇÃO BRASILEIRA DE NORMAS TÉCNICAS, 2011).

A normativa busca padronizar e codificar os elementos e bibliotecas a serem utilizados em projetos e, assim como nos estudos de viabilidade para a construção, de modo que ao ser implementado, através do uso do BIM, seja feito de modo seguro e sem perda de informações durante as fases que envolvem o planejamento até a execução. 
A norma técnica ABNT NBR 15965 consiste em um sistema de classificação das informações relacionadas à padronização do BIM, tendo como parâmetro fornecer informações para indústria da construção, de modo que as nomenclaturas, as informações e os parâmetros sejam utilizados como modelo em todo país. A NBR 15965, quando aplicada ao BIM, deve proporcionar integração ao longo do ciclo de vida, na concepção e na especificação, na orçamentação, no planejamento e no controle, na documentação e no comissionamento (AGÊNCIA,2017).

Como meio de expandir o conceito do BIM, no Brasil, foi instituído em 17 de maio de 2018, o Decreto ํo 9.377, que consiste em instituir a estratégia de disseminação do BIM em todo território nacional, através do Comitê Gestor da Estratégia BIM BR. Por meio do conceito de modelagem, as mudanças irão proporcionar um ambiente adequado de investimento nos setores da construção, incorporado às bibliotecas e normas técnicas específicas para esta adoção (BRASIL..., 2018).

Com a legislação em vigência, instituiu-se a criação da Câmara Brasileira de BIM (CBIM), com intuito de discutir as políticas públicas de implantação e disseminação do BIM no setor da AEC. O CBIM propõe discussões, em âmbito regional e nacional, sobre softwares, contratos, licitações, criação de normas e certificações de processo, quanto à utilização desse conceito BIM em obras públicas (BRASIL, 2018).

Quanto aos meios de propagação da estratégia, o Decreto no 9.377 propõe os seguintes objetivos específicos:

Art. 2ํㅡ A Estratégia BIM BR tem os seguintes objetivos específicos:

I - difundir o BIM e seus benefícios;

II - coordenar a estruturação do setor público para a adoção do BIM;

III - criar condições favoráveis para o investimento, público e privado, em BIM;

IV - estimular a capacitação em BIM;

Disponível em: https://www.nucleodoconhecimento.com.br/engenharia-civil/interoperabilidade-deprojetos 
V - propor atos normativos que estabeleçam parâmetros para as compras e as contratações públicas com uso do BIM;

$\mathrm{VI}$ - desenvolver normas técnicas, guias e protocolos específicos para adoção do BIM;

VII - desenvolver a Plataforma e a Biblioteca Nacional BIM;

VIII - estimular o desenvolvimento e aplicação de novas tecnologias relacionadas ao BIM; e

IX - incentivar a concorrência no mercado por meio de padrões neutros de interoperabilidade BIM. (BRASIL,2018).

Atualmente, entre os objetivos da comissão está a busca por desenvolver um estatuto como forma de institucionalizar a implantação do BIM no Brasil, assim como na divulgação por meio de conferencias e eventos, destacando a importância no mundo acadêmico e na qualificação das pessoas para o uso da ferramenta.

Figura 3 - Resumo do plano de implantação do BIM.

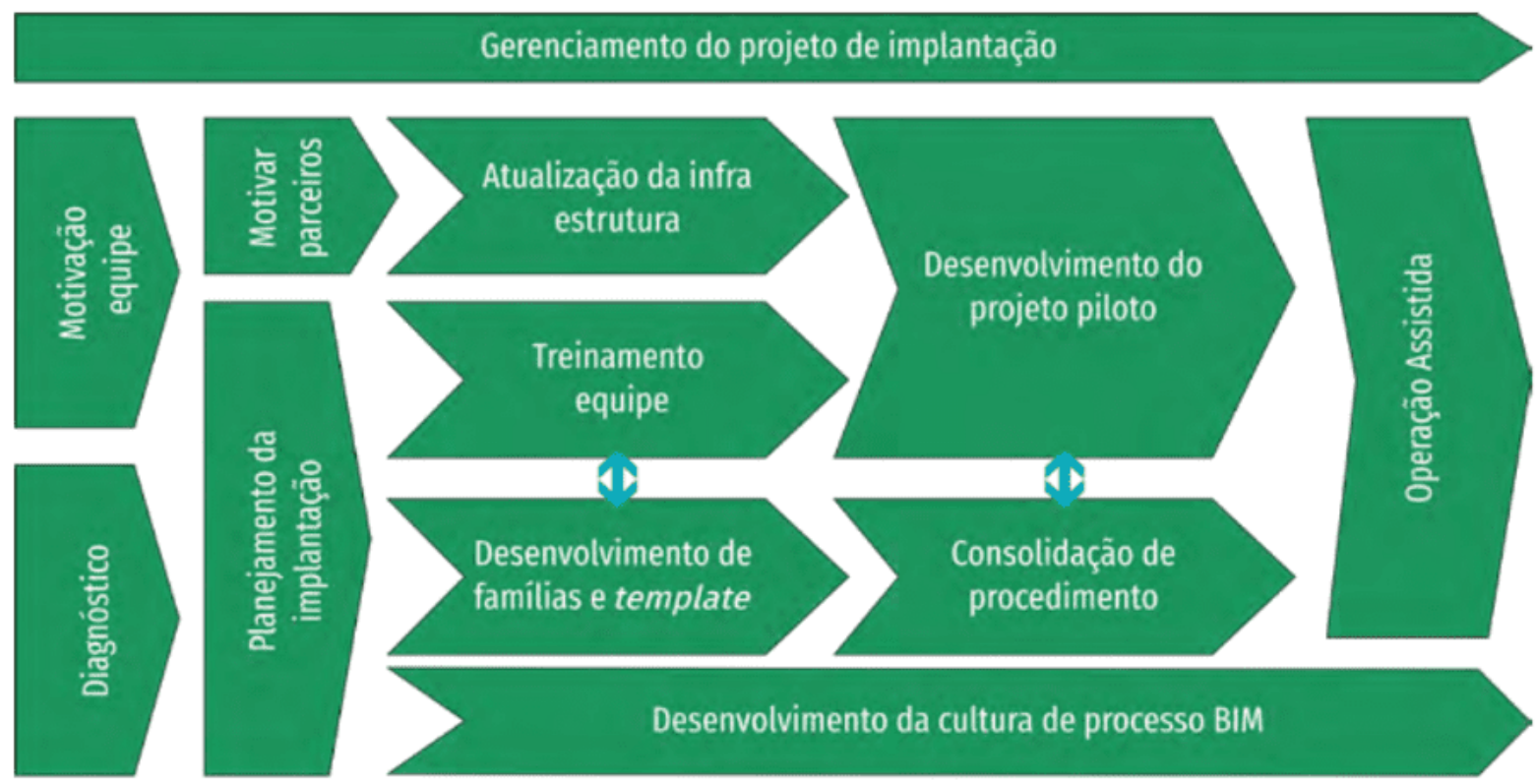

Fonte: AGÊNCIA, 2017.

RC: 45490

Disponível em: https://www.nucleodoconhecimento.com.br/engenharia-civil/interoperabilidade-deprojetos 
A estratégia do setor público consiste em promover por intermédio do conceito BIM, um ambiente adequado ao investimento e difusão no país, tendo como ápice, a exigência da adoção do conceito BIM nos processos de licitação em obras públicas ou no gerenciamento das edificações e infraestrutura que requer adequação da estrutura e dos processos internos. "É necessária a difusão do que é este novo paradigma da indústria da construção e quais benefícios trará para o cidadão brasileiro e para o setor de edificações e infraestrutura" (AGÊNCIA,2017, p. 35).

\section{MATERIAIS E MÉTODOS}

Para este estudo se optou pela estratégia de pesquisa denominada bibliográfica, sendo que "as fontes para a escolha do assunto podem originar-se de experiências pessoal ou profissional, de estudos e leitura, da observação, da descoberta de discrepâncias entre trabalhos ou da analogia com temas de estudo de outras disciplinas ou de áreas científicas" (MARCONI; LAKATOS,2010, p.27).O método de pesquisa consistiu por meio bibliográfico, com o levantamento de referencial teórico, como teses, dissertações, artigos científicos, livros e outras fontes relevantes, contendo subsídios para contextualizar o BIM, sobre os benefícios aplicados na gestão de projetos da AEC relacionado à interoperabilidade de projetos, além de destacar a importância e exemplos para o setor público.

Segundo Marconi; Lakatos (2010, p.65), o método científico consiste em um "conjunto de atividades sistemáticas e racionais, que com maior segurança e economia permite alcançar o objetivo, traçando o caminho a ser seguido, detectando erros e auxiliando as decisões dos cientistas".

\section{RESULTADOS}

Mediante as pesquisas bibliográficas, foi verificado que a adoção do BIM caminha a passos curtos, não apenas pelo alto custo de software e infraestrutura da informação, mas por meio da carência de profissionais capacitados, sendo que muitas empresas optam pela terceirização.

Disponível em: https://www.nucleodoconhecimento.com.br/engenharia-civil/interoperabilidade-deprojetos 
Segundo Barreto et al. (2016), por meio do estudo sobre o "BIM no cenário de arquitetura e construção civil no Brasil", entre as trinta empresas estudadas que utilizaram-se da modelagem da informação, constatou-se que os profissionais das empresas que atuam no setor de arquitetura, o BIM e empregado na finalização dos projetos arquitetônicos da construção, em outros casos, obteve-se dados que comprovam que a maioria das organizações pesquisadas $(41,9 \%)$ utiliza o BIM em todas as fases de todos os projetos. Barreto et al. (2016) afirma que " caso as empresas do setor da construção civil tivessem respondido, as respostas não iriam condizer com a realidade, uma vez que, no Brasil, o BIM é utilizado apenas na parte do projeto, e muito raramente acompanha a obra até o final" (BARRETO, 2016, p.5).

Imagem 5 - Utilização do BIM em projetos do setor privado.

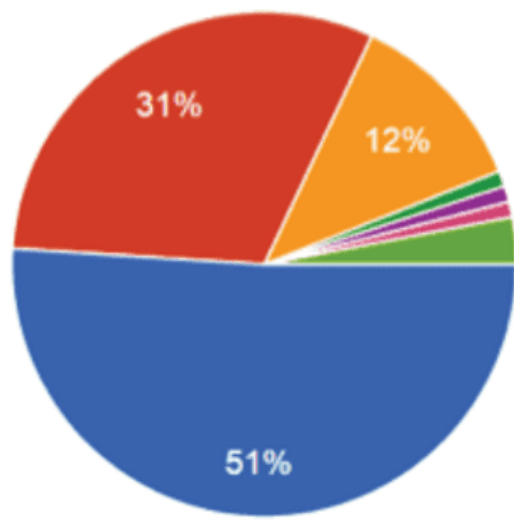

O BIM está presente em menos de $20 \%$ dos projetos 51

O BIM está presente em $20 \%$ dos projetos 31

O BIM está presente em $40 \%$ dos projetos 12

O BIM está presente em $50 \%$ dos projetos

O BIM está presente em $60 \%$ dos projetos

O BIM está presente em $80 \%$ dos projetos

O BIM está presente em $100 \%$ dos projetos

O BIM não está presente

Fonte: Barreto et al., 2016

Com o intuito de verificar o processo de interoperabilidade de projetos, no setor da Administração Pública, não se tornou viável mediante a ausência de projetos que passaram pelo processo de interoperabilidade. No contexto brasileiro, os poderes estaduais estão se organizando de forma individual para adoção do BIM em projetos de empreendimentos públicos e infraestrutura, mediante um comitê estadual, visto ainda o país possuir diretrizes formalizadas quanto ao uso do BIM.

Entretanto, no setor privado, autores referenciam casos de incoerências, em função da falta de interação entre os agentes envolvidos, além da deficiência por meio de práticas gerenciais para um fluxo de trabalho organizado, sendo que um dos fatores

Disponível em: https://www.nucleodoconhecimento.com.br/engenharia-civil/interoperabilidade-deprojetos 
a ser considerado consiste no alto investimento e treinamento para a capacitação da cadeia produtiva, assim como valor para implantação, que em função do alto valor orçado torna o projeto ofertado muito caro, inviabilizando a contratação.

Quanto à interoperabilidade, surge a necessidade de que os softwares de diferentes fornecedores possam ter portabilidade de integração de plataformas IFC e informações, proporcionando a incorporação de sistemas de interoperabilidade adotada de forma adequada. Entretanto, autores informam que ainda ocorrem perdas de informações e na qualidade dos projetos importados neste formato de arquivos, em função da inexistência de um padrão nas propriedades dos componentes, podendo ser apresentados por diferentes aplicativos BIM.

Entre as vantagens da implementação do BIM, nas empresas do setor privado, está o interesse da mudança para redução de falhas na fase de concepção de projetos,disponibilizando o acesso de informações do projeto aos colaboradores em tempo, em um único ambiente que permite múltiplos ciclos de quantificação e esses consistem na mitigação de riscos, auxiliando na escolha de materiais, transparência na informação entre contratante e contratado, planejamento mais eficiente, facilitando a avaliação do ciclo de vida do projeto.

Visando processo de estudo sobre a aplicação ao conceito BIM em projetos de empreendimentos do setor público, a principal contribuição deste trabalho consiste em analisar os benefícios gerados no processo de interoperabilidade na gestão de projetos por meio da ferramenta BIM aplicada ao ramo das engenharias, destacando a metodologia adotada no processo, a viabilidade para as entidades governamentais, além dos desafios encontrados no processo de incentivo de sua adoção, permitindo que os problemas sejam identificados na concepção dos projetos.

\section{CONCLUSÃO}

Este trabalho abordou o estudo sobre a interoperabilidade entre os projetos, através da modelagem das disciplinas e análise de interferências e incoerências com o uso de ferramentas BIM. Outro ponto apresentado foi de dificuldade, que consiste na

Disponível em: https://www.nucleodoconhecimento.com.br/engenharia-civil/interoperabilidade-deprojetos 
ausência de políticas públicas para aumentar a difusão do setor da construção para a adoção das práticas BIM, assim como em projetos públicos.

Acredita-se que para os próximos anos, o BIM possa ser difundido nos setores privados e, principalmente, em obras governamentais, assim como o processo de padronização que já está em desenvolvimento no país.

Como a tecnologia se encontra em fase preliminar no país, em função de pouca abrangência das empresas do setor privado, isso se deve mediante a falta de conhecimento técnico das organizações, necessidade de parâmetros e normativas mais abrangentes, que especifiquem as exigências governamentais, assim como na necessidade de profissionais qualificados.

Os estudos com as instituições públicas e terceirizadas permitiram perceber a ausência de compatibilidade entre os projetos executivos de uma obra, dificultando a análise no setor.

As sugestões futuras para este estudo consistem na tentativa de apresentar, através de um modelo prático, um comparativo com os valores que seriam economizados com a utilização da compatibilização de projetos ou edificação já consolidada.

\section{REFERÊNCIAS}

AGÊNCIA BRASILEIRA DE DESENVOLVIMENTO INDUSTRIAL CLASSIFICAÇÃO DA INFORMAÇÃO NO BIM. GUIA 2 - Classificação da Informação no BIM. Coletânea Guias BIM ABDI-MDIC, Agência Brasileira de Desenvolvimento Industrial. - Brasília, DF, 2017. Vol. 2; 38 p.

ANDRADE, Max Lira Veras X. de; RUSCHEL, Regina Coeli. Interoperabilidade de aplicativos BIM em arquitetura por meio do formato ifc. Revista Gestão \& Tecnologia de Projetos, São Paulo, 01 dezembro 2009, Vol.4(2), p.76-111. 
ASSOCIAÇÃO BRASILEIRA DE NORMAS TÉCNICAS. NBR 15965-1: Sistema de classificação da construção: Terminologia e estrutura. Rio de Janeiro, 2011.

AUTODESK. Bim and the Future of AEC. Disponível em: <https://www.autodesk.com/solutions/bim>. Acesso em: 19 junho de 2018.

BARRETO, B. V; SANCHES, J. L. G; ALMEIDA, T. L. G; RIBEIRO, S. E. C. O Bim no Cenário de Arquitetura e Construção Civil Brasileiro. Disponível em: <http://www.fumec.br/revistas/construindo/article/view/4811/2442 >. Acesso em: 05 junho de 2018.

BUILDING SMART Internacional. Industry Foundation Classes (IFC) - An Introduction. Disponível em: < https://technical.buildingsmart.org/standards/ifc/>. Acesso em: 20 agosto de 2018.

BRASIL. DECRETO № 9.377, DE 17 DE MAIO DE 2018. Institui a Estratégia Nacional de Disseminação do Building Information Modelling. Brasília, DF, maio 2018. Disponível em: <http:/www.planalto.gov.br/ccivil_03/_ato20152018/2018/decreto/D9377.htm>. Acesso em: 12 junho de 2018.

CRESPO, C. C.; RUSCHEL, R. C. R. Ferramentas BIM: um desafio para a melhoria no ciclo de vida do projeto. In: ENCONTRO DE TECNOLOGIA DE INFORMAÇÃO E COMUNICAÇÃO NA CONSTRUÇÃO CIVIL, Porto Alegre, 2007. Anais... Porto Alegre, 2007.

EASTMAN, C. et al. Manual de BIM: um guia de modelagem da informação da construção para arquitetos, engenheiros, gerentes, construtores e incorporadores. Porto Alegre: Bookman, p.01-25, 2013.

KASSEM, Mohamad e AMORIM, Sergio R. Leusin. Building Information Modeling no Brasil e na União Europeia. Ministério do desenvolvimento, indústria e comércio exterior (MDIC). Brasília, 2015. 
KÖCHE, José Carlos. Fundamentos de metodologia científica: teoria da ciência e iniciação à pesquisa. Petrópolis, 34 ed., RJ: Vozes, p.29, 2015.

MARCONI, Maria de Andrade; LAKATOS, Eva Maria. Fundamentos de Metodologia Científica. 7.ed. São Paulo: Atlas, 2010.

MELHADO, Silvio Burrattino. Qualidade do projeto na construção de edifícios: aplicação ao caso das empresas de incorporação e construção. Tese de Doutorado - Escola Politécnica, Universidade de São Paulo. São Paulo, 1994.

MOREIRA, Lorena Claudia de Souza; RUSCHEL, Regina Coeli. Impacto da adoção de BIM em Facility Management: uma classificação. PARC Pesquisa em Arquitetura e Construção, Campinas, SP, v. 6, n. 4, p. 277-290, dez. 2015. Disponível em: $\quad$ <https://periodicos.sbu.unicamp.br/ojs/index.php/parc/article/view/8634982>. Acesso em: 22 junho de 2018.

MUNDO BIM. Softwares BIM: fique por dentro de tudo!. Disponível em: <http://mundobim.com.br/tag/revit/>. Acesso em 30 de junho de 2018.

OLIVEIRA, Chystianne Maria Rodrigues de. Contribuições ao Processo de Projeto de Arquitetura no Setor Público - Um estudo de Caso.São Paulo. 2016.156 f. Dissertação (Mestrado em Arquitetura e Urbanismo) FAUUSP, 2016.

Portal BIM Paraná. BIM nas Obras Públicas. Disponível em:<http://www.bim.pr.gov.br/modules/conteudo/conteudo.php?conteudo=79>. Ace sso em 20 de junho de 2018.

PROJECT MANAGEMENT INSTITUTE - PMI. Um Guia do Conhecimento em Gerenciamento de Projetos (Guia PMBOK). 616 p, 5ed. edição, p.3, 2013.

SANTOS, Eduardo Toledo. Bim bem feito. Revista Estrutura, São Paulo, 3 ed., ano 1, p. 64-65, 2017. 
THOMAZ, Ercio. Tecnologia, gerenciamento e qualidade na construção. $1^{\stackrel{a}{a}}$ ed. São Paulo: PINI, p. 275, 2001.

Enviado: Outubro, 2019.

Aprovado: Janeiro, 2020. 